Ciência Florestal, Santa Maria, v. 24, n. 3, p. 597-607, jul.-set., 2014

ISSN 0103-9954

\title{
INVASÃO BIOLÓGICA DE Corythucha ciliata EM ESPAÇOS VERDES URBANOS DE PORTUGAL: MODELAÇÃO DO NICHO ECOLÓGICO COM O MÉTODO DE MÁXIMA ENTROPIA
}

\author{
BIOLOGICAL INVASION OF Corythucha ciliata IN GREEN URBAN SPACES IN \\ PORTUGAL: A NICHE MODELING APPROACH USING MAXIMUM ENTROPY
}

\author{
Maria Alice da Silva Pinto ${ }^{1}$ Ana Paula Soares Gonçalves ${ }^{2}$ Sónia Alexandra Paiva Santos ${ }^{3}$ \\ Mónica Roldão Lemos de Almeida ${ }^{4}$ João Carlos Martins de Azevedo ${ }^{1}$
}

\begin{abstract}
RESUMO
Corythucha ciliata (Hemiptera: Tingidae) é um inseto nativo da América do Norte que foi introduzido na Europa, pela Itália, em 1964. Desde então tem se expandido por toda a Europa, desconhecendo-se a data de chegada e a sua área de distribuição em Portugal. Esta importante praga invasora alimenta-se na face inferior das folhas de plátano, uma das mais importantes árvores ornamentais nos espaços verdes urbanos em Portugal, causando senescência prematura e eventualmente morte, em casos de infestações severas consecutivas. A modelação de nicho está se tornando uma ferramenta cada vez mais importante na gestão de invasões biológicas, tanto antes como depois da introdução do organismo invasor. Neste estudo, o software MaxEnt (máxima entropia) foi usado na modelação da distribuição invasiva potencial de Corythucha ciliata em Portugal, a partir de um conjunto de variáveis ambientais e de dados de presença do inseto, obtidos a partir da observação de folhas de plátanos amostrados por todo o país. De acordo com o melhor modelo gerado pelo MaxEnt, as áreas de maior adequabilidade potencial à invasão de Corythucha ciliata encontram-se no norte de Portugal apresentando o sul e as regiões de maior altitude do norte e centro adequabilidade reduzida ou nula. Observações laboratoriais da biologia de Corythucha ciliata aliadas aos registos de ausência em várias localidades do sul de Portugal e ocorrência predominante na metade norte de Espanha suportam o modelo desenvolvido. Porém, a validação do modelo requer futuras prospecções nas áreas de reduzida adequabilidade e onde a praga se encontrava virtualmente ausente no momento em que foi realizada a amostragem. Os modelos de adequabilidade podem ser usados como ferramenta auxiliar na tomada de decisão no que concerne à gestão dos espaços verdes.
\end{abstract}

Palavras-chave: MaxEnt; gestão de espaços verdes; espécie invasora; Platanus spp.

\begin{abstract}
Corythucha ciliata (Hemiptera: Tingidae) is an insect native to North America which has been introduced into Europe, through Italy, in 1964. Since then it has expanded across Europe being its date of arrival and distributional range in Portugal unknown. This important invasive pest feeds on the underside of the leaves of sycamore trees, one of the most widespread ornamental tree in urban areas of Portugal, causing their premature senescence and eventually death, in case of consecutive severe infestations. Habitat modeling is becoming an increasingly important tool for managing biological invasions, either prior or

1 Engenheiro Florestal, PhD., Professor Adjunto do Departamento de Ambiente e Recursos Naturais, Centro de Investigação de Montanha (CIMO), Instituto Politécnico de Bragança, Campus de Sta. Apolónia, Apartado 1172, 5301-855 Bragança, Portugal. apinto@ipb.pt / jazevedo@ipb.pt

2 Licenciada em Educação Ambiental, Msc., Centro de Investigação de Montanha (CIMO), Instituto Politécnico de Bragança, Campus de Sta. Apolónia, Apartado 1172, 5301-855 Bragança, Portugal. g.s.ana@hotmail.com

3 Bióloga, Dra ${ }^{a}$, Investigadora Auxiliar do Centro de Investigação de Montanha (CIMO), Instituto Politécnico de Bragança, Campus de Sta. Apolónia, Apartado 1172, 5301-855 Bragança, Portugal. saps@ipb.pt

4 Bióloga, Msc., Rua António José de Almeida, lote 1137A, Quinta do Conde, Sesimbra, Portugal. almeida.monika@gmail.com
\end{abstract}

Recebido para publicação em 4/04/2012 e aceito em 12/04/2013

Ci. Fl., v. 24, n. 3, jul.-set., 2014 
after the introduction of the invasive organism. In this study the software MaxEnt (maximum entropy) was used to model the distribution of Corythucha ciliata in its Portuguese invasive range, from a set of environmental variables and georeferenced occurrence data obtained from observation of Platanus spp. leaves sampled all over the country. According to the best model developed, the areas of greater suitability to invasion of Corythucha ciliata are located in the northern portion of the country whereas the more southern and mountainous areas are of low or virtually null suitability. Laboratory observations of Corythucha ciliata biology allied to records of pest absence across several localities of southern Portugal and predominant occurrence in the northern half of Spain support the model developed. However, model validation requires future prospection in the areas of predicted reduced suitability and where the pest was virtually absent at the moment of sampling. Suitability models can be a useful tool for decision making in management of green spaces.

Keywords: MaxEnt; modeling; invasive pest; Platanus spp.

\section{INTRODUÇÃO}

As invasões biológicas são unanimemente reconhecidas como um dos processos mais significativos de alterações ambientais associadas aos humanos à escala global e como a mais importante ameaça à biodiversidade a seguir à destruição de habitat. Apesar dos prejuízos econômicos e ambientais serem mais importantes nos ecossistemas naturais (PIMENTEL, 2011), as invasões biológicas dos ecossistemas urbanos podem também ter impactos dramáticos. Um dos casos mais paradigmáticos foi a invasão do fungo causador da grafiose, Ophiostoma novoulmi (Brasier), do ulmeiro (Ulmus spp.) que em pouco mais de 20 anos levou praticamente ao desaparecimento desta árvore em toda a Europa e América do Norte (BRASIER, 1991). Esta invasão alterou profundamente a fisionomia e as funções dos espaços verdes de muitos centros urbanos, pois o ulmeiro era uma das espécies ornamentais mais comuns tanto na Europa como na América do Norte (CAMPANELLA, 2003). Para além dos prejuízos que podem causar nos espaços verdes urbanos, existe o risco das invasões biológicas se propagarem às florestas naturais, como foi recentemente documentado nos EUA (DODDS e ORWIG, 2011) para Anoplophora glabripennis (Motschulsky) (Coleoptera: Cerambycidae), considerada uma das 100 espécies invasoras mais perigosas a nível mundial (SIMBERLOFF e REJMÁNEK, 2011).

As pragas e doenças exóticas encontram nas árvores dos espaços verdes urbanos (ruas, parques, florestas) condições particularmente favoráveis ao processo invasivo, por duas razões principais. Primeiro, porque as árvores que crescem em ambiente urbano estão frequentemente expostas a inúmeros fatores de estresse (ex: poluição, compactação do solo, limitação do espaço para crescimento, baixa disponibilidade de água resultante da impermeabilização do solo e vandalismo) capazes de causar desequilíbrios fisiológicos que facilitam as fases de estabelecimento e persistência do processo invasivo. Segundo, porque a presença humana auxilia as fases de introdução e expansão através do transporte direto do invasor entre regiões ou continentes ou através das vias de comunicação que funcionam muitas vezes como importantes corredores de dispersão a uma escala mais local.

Uma das consequências negativas da globalização é a ameaça permanente da introdução e subsequente propagação de organismos invasores. $\mathrm{Na}$ extensa lista de organismos invasores presentes na Europa, disponibilizada no portal DAISI (2011), European Invasive Alien Species Gateway, encontrase a espécie focal deste estudo: Corythucha ciliata (Say 1832) (Hemiptera: Tingidae). Corythucha ciliata é uma espécie nativa da América do Norte que se encontra atualmente difundida e estabelecida por toda a Europa e também em outras partes do Mundo, tais como China (JU et al., 2009), Japão (TOKIHIRO et al., 2003), Chile (PRADO, 1990) e Austrália (DOMINIAK et al., 2008).

Na Europa, Corythucha ciliata foi observada pela primeira vez atacando plátanos (Platanus spp.) da cidade de Pádua, Itália, em 1964 (SERVADEI, 1966). Em menos de 20 anos, após a deteção na Itália, esta espécie já se encontrava por todo o centro e sul da Europa (MACELJSKI, 1986) e atualmente há registos em praticamente todos os países do subcontinente (DAISI). Na vizinha Espanha, a primeira observação foi feita em Gerona, em 1982, tendo-se subsequentemente difundido pelo menos na metade norte do país (ROMANYK e CADAHIA, 
2003, e numerosas referências em GROSSO-SILVA e AGUIAR, 2007). Desconhece-se a data exata de introdução em Portugal sendo o primeiro registo oficial de 2007 (GROSSO-SILVA e AGUIAR, 2007). Estes autores identificaram a praga em folhas de plátano colhidas na zona metropolitana do Porto e mais a sul, em Rio Maior, desconhecendo-se, até a data deste estudo, a distribuição da praga no resto do país.

Corythucha ciliata é um inseto picadorsugador que se alimenta na face inferior das folhas de plátano causando necroses, primeiro ao longo das nervuras e mais tarde em toda a folha. As folhas ficam amareladas e podem cair prematuramente. As árvores atacadas crescem pouco, ficam debilitadas e, consequentemente, mais suscetíveis ao ataque de outras pragas e doenças, e sofrem uma redução do valor ornamental. Quando as infestações são muito severas, consecutivas e associadas a outros fatores podem até causar a morte das árvores. As infestações são preocupantes quando o inseto transporta o fungo Ceratocystis fimbriata f. platani (ELLIS e HALSTED) Walter que tem causado a morte dos plátanos em países como Itália, França e Suiça (MATASCI e GESSLER, 1997). Para além dos estragos causados nas árvores, quando os níveis de infestação são elevados, os insetos podem invadir edifícios, parques e jardins afetando a utilização plena destes espaços de recreio e lazer pelas pessoas.

O plátano, especialmente a espécie híbrida Platanus acerifolia (Platanus orientalis x Platanus occidentalis), vulgarmente designada por plátano europeu ou plátano de Londres, é uma árvore comum em muitas áreas urbanas de todo o Mundo, devido ao elevado grau de adaptação ao ambiente urbano e valor estético. A sua abundância em Londres, por exemplo, está na origem do nome comum "London plane". Para além de Londres, esta espécie é um elemento dominante dos espaços verdes em toda a Europa, especialmente nos países do sul, como é o caso de Portugal. Assim, se as condições ambientais forem favoráveis ao estabelecimento e persistência de Corythucha ciliata, o seu impacto nos espaços verdes das áreas urbanas em Portugal pode ser significativo.

A distribuição geográfica de muitas espécies de plantas e animais, nativas ou exóticas, pode ser prevista através da utilização de modelos ecológicos de espécies (GUISAN e THUILLER, 2005; ELITH et al., 2006). Para além do seu uso na predição dos impactos potenciais das alterações globais no risco de extinção de espécies (THOMAS et al.,
2004; THUILLER et al., 2005a), no planeamento de reservas (WILSON et al., 2005; MARINI et al., 2009), ou na identificação de áreas de distribuição desconhecida de espécies raras (GUISAN et al., 2006), os modelos ecológicos de espécies têm se revelado muito úteis na previsão do risco de invasões biológicas e, consequentemente, na sua gestão (PETERSON e VIEGLAIS, 2001; PETERSON, 2003; PETERSON et al., 2003; THUILLER et al., 2005b; CHEN et al., 2007; FICETOLA et al., 2007; ROURA-PASCUAL et al., 2009; VILLEMANT et al., 2011). Os modelos podem ser usados tanto na avaliação pró-ativa, antes da introdução do invasor, como no desenvolvimento de medidas de controle da invasão após introdução.

Neste estudo recorreu-se ao método da máxima entropia implementado pelo software MaxEnt (PHILLIPS et al., 2006), para modelar a distribuição geográfica potencial de Corythucha ciliata em Portugal continental de forma a avaliarse as áreas de maior adequabilidade a esta praga invasora. Com base no princípio da máxima entropia, seguiu-se uma abordagem de modelação de nicho ecológico (ecological niche modelling) a qual permite associar ao espaço probabilidades que representam a adequabilidade do habitat de uma espéciee desta forma estimara suaárea de distribuição geográfica potencial (nicho fundamental), de acordo com condicionantes estabelecidas a partir dos níveis das variáveis ambientais amostradas nos locais de observação dessa espécie (PHILLIPS et al., 2004; PHILLIPS et al., 2006).

\section{MATERIAL E MÉTODOS}

\section{Área de estudo e amostragem}

A área de estudo deste trabalho é o território continental de Portugal, para o qual se modelou a distribuição potencial de Corythucha ciliata. Para a obtenção de dados de presença foram apenas consideradas as áreas de ocorrência de plátanos (Platanus spp.), uma vez que Corythucha ciliata é uma praga especifica desta árvore. Estas áreas correspondem essencialmente a ambientes urbanos onde o plátano é uma das árvores mais utilizadas em vias públicas, parques, rotatórias, e outros espaços verdes.

A informação sobre presença/ pseudoausência de Corythucha ciliata foi obtida por observação de folhas de plátano recolhidas por todo o país. No período de Junho a Outubro de 2009 foram 
efetuadas prospeções sistemáticas pelos autores do trabalho, principalmente em áreas urbanas da zona norte do país, e por colaboradores distribuídos pelas restantes regiões. Os colaboradores seguiram o seguinte procedimento: (i) observaram árvores em vilas e cidades da sua região, com base em documentos de identificação da praga e da árvore fornecidos pelos autores; (ii) recolheram material que foi posteriormente enviado para confirmação da praga em laboratório (presença de adultos, ninfas e/ou excrementos) e (iii) registaram a localização (através do nome da rua, praça ou outros elementos) das árvores para posterior obtenção de coordenadas geográficas recorrendo ao Google Maps (2011). No total prospetaram-se 80 locais representados na Figura 1. Considera-se que a intensidade de amostragem se encontra distribuída pela área de

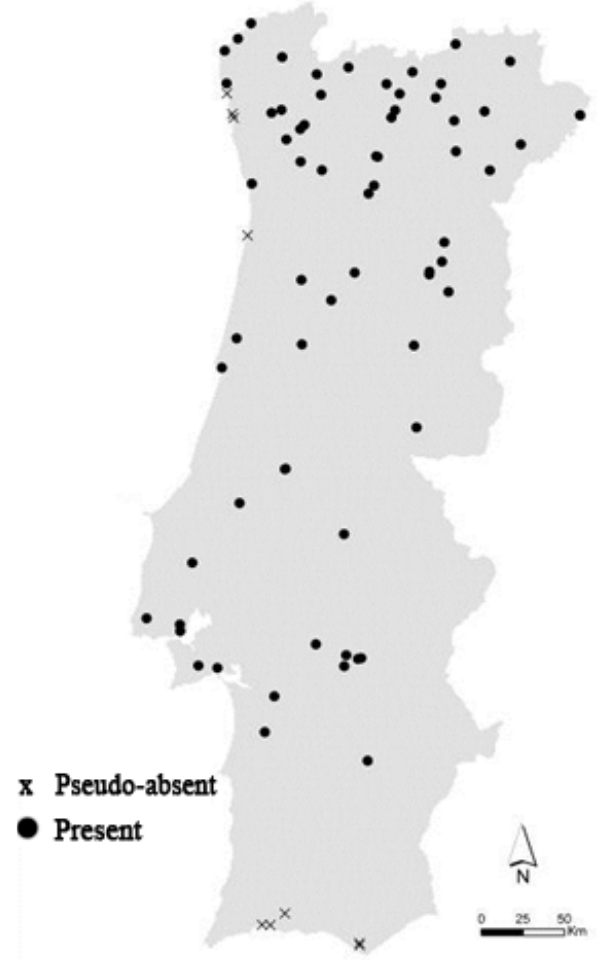

FIGURA 1:Locais de prospeção de Corythucha ciliata em Portugal Continental durante 2009. Os locais de pseudoausência do inseto, que não são utilizados na metodologia seguida neste trabalho, são aqui apresentados com fins meramente ilustrativos.

FIGURE 1: Prospection sites of Corythucha ciliata in continental Portugal during 2009. Absence locations were not used in the method followed here but are shown for illustration purposes only. estudo de forma homogênea. A amostra representa pelo menos cerca de $11 \%$ de todas as cidades (158) e vilas (582) de Portugal uma vez que não é garantido que em todas haja plátanos.

\section{Variáveis ambientais}

Utilizaram-se dados espaciais da distribuição de um conjunto de variáveis climáticas, bioclimáticas e de elevação, obtidos a partir da base de dados WorldClim (2011) em formato matricial (sistema de coordenadas geográficas - Latitude/Longitude, Datum WGS84, resolução de 30 segundos). Foram consideradas no total 27 variáveis, incluindo todas as variáveis bioclimáticas (designadas por "bio" no WorldClim) disponibilizadas na base de dados assim como algumas relativas a temperaturas médias, mínimas e máximas de meses específicos (Tabela 1).

\section{Modelação}

A modelação da distribuição de Corythucha ciliata pelo método da máxima entropia foi efetuada com o software MaxEnt (v.3.3.1) escrito por Steven Phillips, Miro Dudík e Rob Schapire (PHILLIPS et al., 2006; PHILLIPS e DUDÍK, 2008). Esta abordagem requer somente dados de presença e informação ambiental (variáveis ambientais e respetivas transformações, features cujos valores esperados servem de condicionantes do nicho da espécie). A modelação do habitat foi feita com $75 \%$ dos pontos da amostra (designados por dados de treino) permanecendo como dados de teste $25 \%$ das ocorrências selecionadas aleatoriamente pelo MaxEnt. Na modelação utilizaram-se os seguintes parâmetros: convergence threshold $=10^{-5}$; maximum iteration $\mathrm{s}=2000$; features $=$ auto; regularization multiplier $=0,1$; output $=$ cumulative e logistics e a opção add samples to background ativada. Foram inicialmente consideradas no modelo todas as variáveis descriminadas na Tabela 1 (e respetivas transformações, operadas de forma automática). Adicionalmente, testaram-se combinações diversas do menor número possível de variáveis, com o intuito de construir um modelo mais simples que fosse ao mesmo tempo facilmente interpretável e que apresentasse menos erros associados ao eventual efeito de correlação entre variáveis. 
TABELA 1: Variáveis utilizadas na modelação da área de distribuição potencial de Corythucha ciliata. Fonte: WorldClim (2011).

TABLE 1: Variables used in modelling the potencial distribution area of Corythucha ciliata. Source: WorldClim (2011).

\begin{tabular}{|c|c|}
\hline Variável & Variável \\
\hline bio1: temperatura média anual $\left({ }^{\circ} \mathrm{C}\right)$ & bio14: precipitação do mês mais seco \\
\hline bio2: amplitude diurna média $\left({ }^{\circ} \mathrm{C}\right)$ & bio15: sazonalidade da precip. (coeficiente de variação) \\
\hline bio3: isoltermalidade (bio2/bio7) $(* 100)$ & bio16: precipitação do trimestre mais úmido (mm) \\
\hline bio4: sazonalidade da temperatura (desvio padrão $* 100$ ) & bio17: precipitação do trimestre mais seco (mm) \\
\hline bio5: temperatura máxima do mês mais quente $\left({ }^{\circ} \mathrm{C}\right)$ & bio18: precipitação do trimestre mais quente (mm) \\
\hline bio6: temperatura mínima do mês mais frio $\left({ }^{\circ} \mathrm{C}\right)$ & bio19: precipitação do trimestre mais frio (mm) \\
\hline bio7: amplitude térmica anual (BIO5-BIO6) $\left({ }^{\circ} \mathrm{C}\right)$ & tmean6: temperatura média de junho $\left({ }^{\circ} \mathrm{C} * 10\right)$ \\
\hline bio8: temperatura média do trimestre mais úmido $\left({ }^{\circ} \mathrm{C}\right)$ & tmean7: temperatura média de julho $\left({ }^{\circ} \mathrm{C} * 10\right)$ \\
\hline bio9: temperatura média do trimestre mais seco $\left({ }^{\circ} \mathrm{C}\right)$ & tmean8: temperatura média de agosto $\left({ }^{\circ} \mathrm{C} * 10\right)$ \\
\hline bio10: temperatura média do trimestre mais quente $\left({ }^{\circ} \mathrm{C}\right)$ & tmean9: temperatura média de setembro $\left({ }^{\circ} \mathrm{C} * 10\right)$ \\
\hline bio11: temperatura média do trimestre mais frio $\left({ }^{\circ} \mathrm{C}\right)$ & tmin5: temperatura mínima do mês de maio $\left({ }^{\circ} \mathrm{C} * 10\right)$ \\
\hline bio12: precipitação anual (mm) & tmax5: temperatura máxima do mês de maio $\left({ }^{\circ} \mathrm{C} * 10\right)$ \\
\hline bio13: precipitação do mês mais úmido & alt: altitude (m) \\
\hline
\end{tabular}

\section{Avaliação do modelo}

O modelo foi avaliado com base na variação da taxa de erros de omissão no conjunto de dados de teste em função de um limite de decisão (threshold) de adequabilidade e na curva ROC (Receiver Operating Characteristic). A percentagem de erros de omissão dos dados de teste deve variar com o limite de decisão cumulativo de forma linear e qualquer afastamento deste pressuposto permite avaliar o desempenho do modelo. Pode ser ainda calculado um parâmetro estatístico (teste binomial) para testar a hipótese nula de que o modelo não prediz os dados de teste melhor do que se a escolha fosse completamente aleatória para a mesma percentagem de área predita. A curva ROC relaciona a sensibilidade da classificação (proporção de localizações com presença de Corythucha ciliata classificadas corretamente pelo modelo $=1$ - erro de omissão) com a percentagem de área predita (proporção de todas as células da matriz preditas como adequadas para a espécie $=1$ - especificidade) em substituição de erros de comissão, uma vez que estes não podem ser avaliados sem dados de ausência (PHILLIPS et al., 2006) para todos os valores de limites possíveis. A AUC (Area Under Curve) corresponde à área no gráfico por baixo da curva ROC, tanto para os dados de treino como de teste, e proporciona uma medida quantitativa standard do desempenho do modelo. Quanto maior o valor da AUC, melhor o modelo. Uma predição perfeita teria um valor de AUC de 1 (porém, quando se trabalha com dados apenas de presença, o máximo valor atingido é menor que 1), uma boa predição corresponde a 0,75 , e um modelo aleatório corresponde a 0,5 (PHILLIPS et al., 2004, 2006). Foi ainda analisada a contribuição das diferentes variáveis no ganho regularizado do modelo, quando omitidas e quando modeladas isoladamente. Mais detalhes da avaliação de modelos de distribuição com base na máxima entropia podem ser consultados em Phillips et al. (2004, 2006), Phillips e Dudík (2008) e Elith et al. (2011).

\section{RESULTADOS E DISCUSSÃO}

Este estudo constituiu a primeira prospeção sistemática de Corythucha ciliata de abrangência nacional. Dos 80 locais prospetados, Corythucha ciliata foi observada em 71 e em 9 não se encontrou qualquer sinal indicador da sua presença (Figura 1). Todos os pontos de ausência foram detectados em localidades junto à costa, 5 no sul (distrito de Faro) e 4 no norte (um no distrito de Viana do Castelo, dois no distrito de Braga, e um no distrito de Aveiro). Uma vez que MaxEnt requer somente dados de presença, na construção do modelo de predição foram utilizados apenas os 71 pontos e as variáveis ambientais da Tabela 1. As tentativas efetuadas com MaxEnt com base na seleção e na combinação 
das 26 variáveis ambientais que teoricamente mais poderiam contribuir para a definição do nicho ecológico resultaram em modelos de capacidade preditiva geralmente elevada. Contudo, a combinação que apresentou o melhor desempenho considera apenas as 17 variáveis descriminadas na Tabela 2.

A precipitação do trimestre mais quente foi a variável que mais contribuiu para o modelo $(14,0 \%)$, seguida da temperatura média de setembro $(10,8 \%)$, temperatura média do trimestre mais quente $(10,3 \%)$, precipitação do trimestre mais úmido $(10,1 \%)$ e temperatura média anual $(9,2 \%)$. Estas variáveis têm uma contribuição mais forte $(54,4 \%)$ do que as restantes relativas à temperatura. Porém, mesmo as variáveis com a contribuição mais fraca (ex: temperatura máxima de maio e temperatura média de junho) revelaram-se importantes, pois a sua remoção reduz a capacidade preditiva do modelo. Tal como acontece com a generalidade dos insetos, o período de maior actividade de Corythucha ciliata, que ocorre entre o final da primavera (maio/ junho) e princípio do outono (setembro/outubro), coincide com a época mais quente do ano. Durante o inverno, o adulto encontra-se hibernando debaixo da casca dos plátanos estando por isso menos exposto aos rigores do clima. Assim, como seria de se esperar, as variáveis ambientais com maior contribuição $(65,7 \%)$ na modelação do nicho de Corythucha ciliata estão diretamente relacionadas com as temperaturas de Verão.

A aplicação dos diferentes critérios na avaliação do modelo obtido mostra que as predições efetuadas foram significativamente superiores às esperadas de um processo aleatório de atribuição de adequabilidade às células do mapa da área de estudo (Tabela 3). O teste binomial de omissão apresentou valores muito significativos $(\mathrm{p}<0,01)$ para todos os limites de decisão testados exceto um em que é significativo $(\mathrm{p}<0,05)$. A taxa de omissão foi de zero para todos os casos, exceto para três em que foi igual a $9,8 \%$ (Tabela 3 ).

O valor da AUC para os dados de treino foi de 0,956 e para os dados de teste de 0,744 (Figura 2). O primeiro valor, aproximando-se de um, indica uma elevada capacidade preditiva do modelo. O segundo valor, resultado particularmente importante porque testa a predição do modelo num conjunto de dados independentes não utilizados na sua construção, é também relevante. Por um lado,

TABELA 2: Contribuição percentual das variáveis utilizadas na construção do modelo com MaxEnt.

TABLE 2: Percent contribution of variables used in model construction with MaxEnt.

\begin{tabular}{lc}
\hline \multicolumn{1}{c}{ Variável } & $\begin{array}{c}\text { Contribuição } \\
\text { percentual }\end{array}$ \\
\hline Precipitação do trimestre mais quente & 14,0 \\
Temperatura média de setembro & 10,8 \\
Temperatura média do trimestre mais quente & 10,3 \\
Precipitação do trimestre mais úmido & 10,1 \\
Temperatura média anual & 9,2 \\
Temperatura mínima do mês de maio & 7,9 \\
Altitude & 7,5 \\
Amplitude térmica anual & 7,4 \\
Temperatura mínima do mês mais frio & 5,3 \\
Temperatura máxima do mês mais quente & 4,9 \\
Precipitação anual & 2,6 \\
Temperatura média do trimestre mais frio & 2,5 \\
Temperatura mínima do mês de maio & 2,4 \\
Temperatura média de agosto & 2,2 \\
Temperatura média de julho & 1,9 \\
Temperatura máxima do mês de maio & 0,6 \\
Temperatura média de junho & 0,3 \\
\hline
\end{tabular}


TABELA 3: Resultados da avaliação do erro de omissão para diferentes limites de decisão.

TABLE 3: Results of the omission error test for different thresholds.

\begin{tabular}{|c|c|c|c|c|c|c|}
\hline $\begin{array}{l}\text { Limite de } \\
\text { decisão } \\
\text { cumulativo }\end{array}$ & $\begin{array}{l}\text { Limite de } \\
\text { decisão } \\
\text { logístico }\end{array}$ & Descrição & $\begin{array}{l}\text { Fração da } \\
\text { área predita }\end{array}$ & $\begin{array}{l}\text { Taxa de } \\
\text { omissão } \\
\text { (treino) } \\
\end{array}$ & $\begin{array}{c}\text { Taxa de } \\
\text { omissão } \\
\text { (teste) }\end{array}$ & $\begin{array}{l}\text { Valor } \\
\text { de P }\end{array}$ \\
\hline 1,000 & 0,034 & Valor cumulativo fixo e igual a 1 & 0,604 & 0,000 & 0,176 & $4,987 \mathrm{E}-2$ \\
\hline 5,000 & 0,107 & Valor cumulativo fixo e igual a 5 & 0,430 & 0,000 & 0,235 & $5,337 \mathrm{E}-3$ \\
\hline 10,000 & 0,171 & Valor cumulativo fixo e igual a 10 & 0,331 & 0,000 & 0,294 & $1,729 \mathrm{E}-3$ \\
\hline 13,356 & 0,206 & Presença de treino mínima & 0,285 & 0,000 & 0,412 & $8,647 \mathrm{E}-3$ \\
\hline 44,248 & 0,482 & Percentil 10 de presença de treino & 0,091 & 0,098 & 0,588 & $4,261 \mathrm{E}-4$ \\
\hline 42,104 & 0,466 & $\begin{array}{c}\text { Sensibilidade e especificidade de treino } \\
\text { iguais }\end{array}$ & 0,098 & 0,098 & 0,588 & $6,934 \mathrm{E}-4$ \\
\hline 44,248 & 0,482 & $\begin{array}{l}\text { Sensibilidade e especificidade de treino } \\
\text { máximas }\end{array}$ & 0,091 & 0,098 & 0,588 & $4,261 \mathrm{E}-4$ \\
\hline 10,628 & 0,178 & $\begin{array}{l}\text { Sensibilidade e especificidade de teste } \\
\text { iguais }\end{array}$ & 0,321 & 0,000 & 0,294 & $1,3 \mathrm{E}-3$ \\
\hline 10,628 & 0,178 & $\begin{array}{l}\text { Sensibilidade e especificidade de teste } \\
\text { máximas }\end{array}$ & 0,321 & 0,000 & 0,294 & $1,3 \mathrm{E}-3$ \\
\hline
\end{tabular}

o valor de 0,744 afasta-se consideravelmente do valor da AUC relativo a predições aleatórias $(0,5)$. Por outro, a proximidade ao valor de 0,75 é também indicadora da boa capacidade preditiva do modelo (PHILLIPS et al., 2004). Considerando ainda que o valor máximo que este teste pode assumir é inferior a $1(0,872$ neste caso $)$ e que a AUC varia com a amplitude natural do nicho das espécies (PHILLIPS et al., 2004), os indicadores avaliados sugerem que o modelo é satisfatório na estimação da distribuição da adequabilidade do habitat potencial de Corythucha ciliata.

O melhor modelo de distribuição da adequabilidade do habitat de Corythucha ciliata

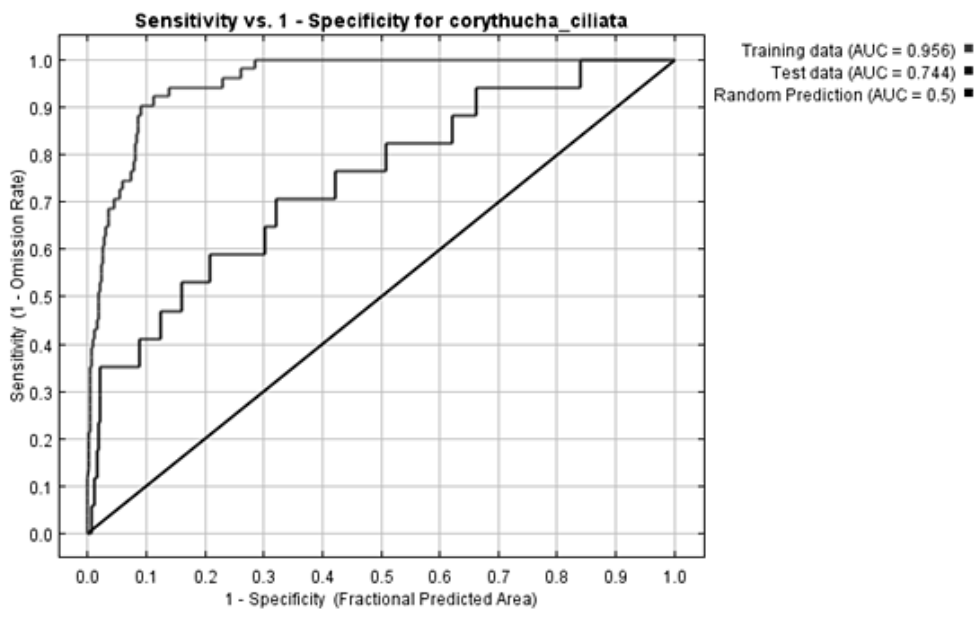

FIGURA 2: Curva ROC (receiver operating characteristic) para dados de treino (curva superior), de teste (intermédia) e de predição aleatória (inferior). A área sob a curva (AUC-area under the curve) mede a capacidade preditiva do modelo. Valores elevados de AUC (perto de 1) indicam erro de omissão reduzido para qualquer limite de decisão.

FIGURE 2: Receiver operating characteristic (ROC) curve for training data (upper curve), test data (intermediate) and random prediction values (lower). AUC (area under curve) measures the predictive capability of the model. High AUC values indicate low omission error rates for different thresholds. 
desenvolvido com MaxEnt é exibido nos mapas da Figura 3. A representação contínua da predição do habitat de Corythucha ciliata numa escala de 0 a 100 (valores cumulativos) mostra que as áreas de adequabilidade mais elevada se situam no norte e centro e as de adequabilidade baixa a muito baixa se situam no sul. Nas áreas do norte e centro, no entanto, detectam-se áreas de baixa ou nula adequabilidade que correspondem aos maciços montanhosos do Noroeste (Serras do Gerês e Barroso), do Nordeste (Serras de Montesinho e Nogueira) e da Serra da Estrela no centro. Apesar da dimensão reduzida, Portugal possui uma heterogeneidade climática muito acentuada que distingue o sul, de influência mediterrânica e por isso mais quente e seco, do norte de influência mais atlântica e por isso mais
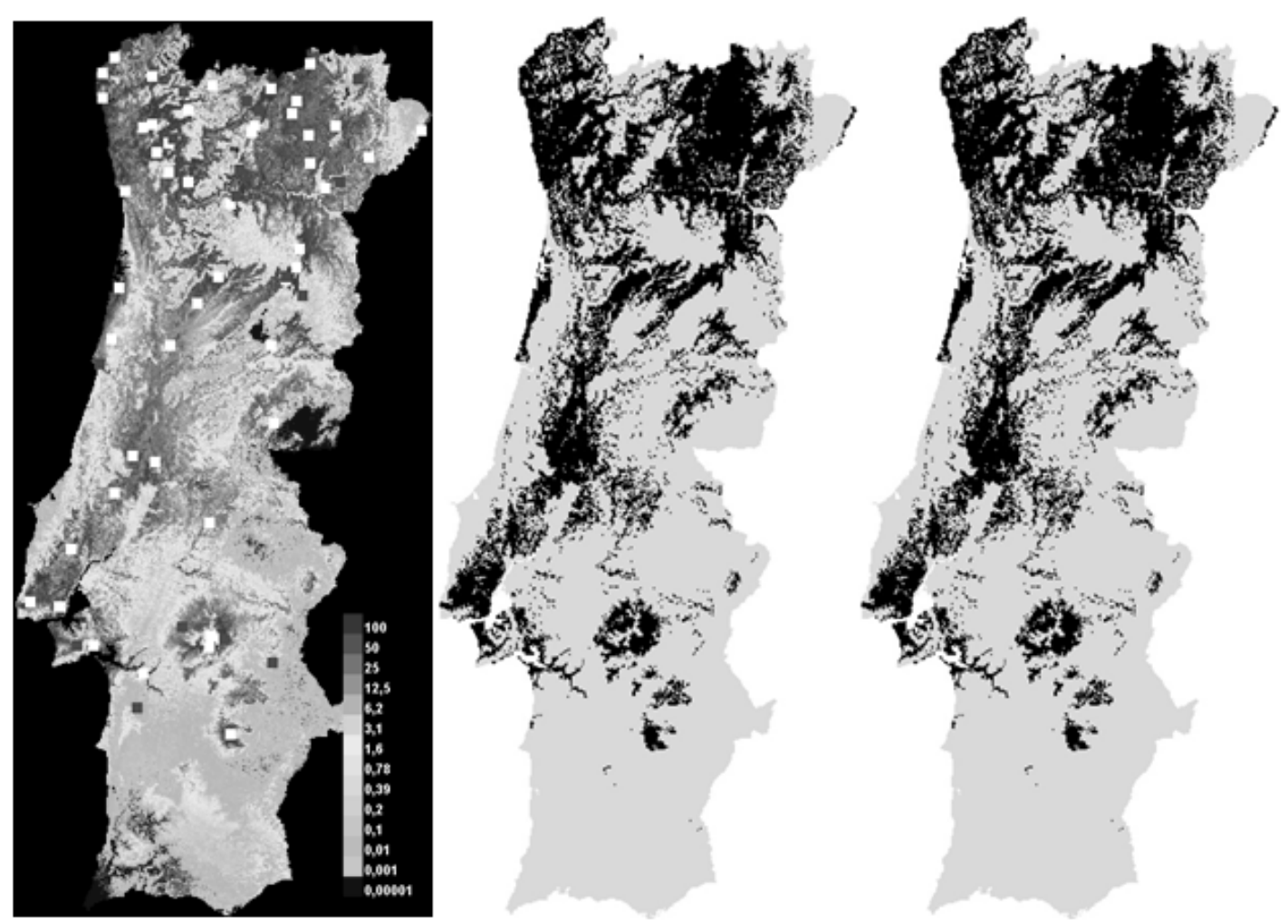

FIGURA 3:Representação da adequabilidade do habitat de Corythucha ciliata em Portugal continental com base em MaxEnt. Esquerda: output cumulativo (escala de 0 a 100); tons escuros indicam melhor adequabilidade; quadrados indicam a localização dos dados de presença: brancos, os dados de treino e escuros os dados de teste; notar que a escala utilizada é logarítmica. Centro: output logístico (corte na distribuição de probabilidades de acordo com o limite de decisão correspondente a minimum training presence $=0,206$ ); células em negro indicam habitat adequado e células em cinza habitat não adequado. Direita: output logístico (corte na distribuição de probabilidades de acordo com o limite de decisão correspondente a equal training sensitivity and specificity $=0,466$ ); células em negro indicam habitat adequado e células em cinza habitat não adequado.

FIGURE 3: Representation of habitat suitability for Corythucha ciliata in Portugal based upon MaxEnt. Left: cumulative output; darker tones indicate higher suitability; squares indicate location of presence data: training data in white and test data in dark; notice that scale is logarithmic. Centre: logistic output (cut in probability distribution according to the minimum training presence threshold $(0,206)$; cells in black indicate suitable habitat and cells in gray non-suitable habitat. Right: logistic output (cut in probability distribution according to the equal training sensitivity and specificity threshold $(0,466)$; cells in black indicate suitable habitat and cells in gray non-suitable habitat. 
úmido e com temperaturas mais amenas no Verão. Ensaios laboratoriais mostram que a fecundidade máxima e a temperatura ótima de desenvolvimento de Corythucha ciliata ocorrem aos $30^{\circ} \mathrm{C}$ e que para valores superiores a $36^{\circ} \mathrm{C}$, e em condições de $80 \%$ de úmidade, o inseto não completa o seu ciclo de desenvolvimento (JU et al., 2011). Estas observações laboratoriais da biologia de Corythucha ciliata aliadas aos registos de ausência em várias localidades do distrito mais ao sul de Portugal (Figura 1) e à ocorrência predominante na metade norte de Espanha (ROMANYK e CADAHIA, 2003; GROSSO-SILVA e AGUIAR, 2007) suportam a predição de menor adequabilidade de habitat obtida para o sul de Portugal.

É importante salientar que o modelo desenvolvido representa o nicho fundamental de Corythucha ciliata e não o nicho realizado, o qual resulta da intercepção da adequabilidade determinada pelos fatores abióticos, da presença do complexo de competidores e inimigos naturais (predadores, parasitoides, doenças) e da disponibilidade de hospedeiro. Ou seja, o modelo pode prever elevada adequabilidade de habitat, mas na ausência de hospedeiro não se verifica a ocorrência de Corythucha ciliata. Por outro lado, as áreas de adequabilidade baixa ou nula não representam ausência absoluta do inseto; ele pode estar presente, mas, devido a limitações de natureza ambiental, a densidade populacional dificilmente atingirá níveis elevados.

\section{CONCLUSÕES}

Este trabalho constitui a primeira prospecção à escala nacional da ocorrência de Corythucha ciliata, uma das pragas invasoras mais importantes dos espaços verdes urbanos da Europa. Desconhece-se a data de introdução de Corythucha ciliata em Portugal. Assim, a fase do processo de invasão (introdução, estabelecimento, expansão e equilibrio na comunidade) em que a espécie se encontra é incerta. É possível que esteja na fase de expansão e que possa ainda se estender aos locais onde estava ausente no momento da recolha de dados. No entanto, a hipótese deste trabalho é a de que a fase de expansão encontra-se praticamente terminada e foi atingido o limite sul da distribuição em Portugal. Esta hipótese poderá ser confirmada em posteriores trabalhos de monitorização de Corythucha ciliata.

Os dados de presença permitiram o desenvolvimento do modelo de habitat potencial para Portugal, o qual genericamente prevê menor adequabilidade nas regiões de maior altitude e no sul do país. O modelo pode ser usado como ferramenta auxiliar na tomada de decisão no que concerne à gestão dos espaços verdes, designadamente no processo de seleção de espécies a utilizar em novos projetos de arborização urbana. Assim, nas áreas de elevada adequabilidade, localizadas no norte, a utilização massiva de plátanos, tal como hoje se verifica, deve ser repensada, uma vez que não existem métodos de tratamento eficazes disponíveis em Portugal para controlar esta praga invasora.

\section{AGRADECIMENTOS}

Este estudo não teria sido possível sem a colaboração generosa de colegas e amigos, que contribuíram com dados de presença/pseudoausência e amostras de folhas de plátano e da praga, nomeadamente: Ana Marta Teodósio, Fernando Rei, Gabriel Figueiredo, Isabel Patanita, João Paulo Castro, João Pedro Pereira, João Rua, João Silva, Jorge Mata Pires, Luís Caparica, Luís Freitas, Luís Nunes, Manuel Beça, Manuel Feliciano, Manuel Fernandes, Marco Duarte, Maria Helena Caixinha, Maria João Videira, Marília Claro, Marinha Esteves, Sérgio Deusdado, Tatiana Ventura. Agradecemos também a Laura Miller pelos esclarecimentos e dados de distribuição de Corythucha ciliata nos EUA. Por último, agradecemos a dois revisores anônimos pelas sugestões que ajudaram a melhorar o artigo.

\section{REFERÊNCIAS BIBLIOGRÁFICAS}

BRASIER, C. M. Ophiostoma novo-ulmi sp. nov., causative agent of the current Dutch elm disease pandemics. Mycopathologia, v. 115, n. 3, p. 151-161, 1991.

CAMPANELLA, T. J. Republic of shade: New England and the American elm. New Have: Yale University Press, 2003.

CHEN, P., WILEY, E. O., MCNYSET, K. M. Ecological niche modeling as a predictive tool: silver and bighead carps in North America. Biological Invasions, v. 9, n. 1, p. 43-51, 2007.

DAISI - European Invasive Alien Species Gateway. Disponível: <http://www.europe-aliens. org >. Acesso em: 24/11/2011.

DODDS, K. J.; ORWIG, D. A. An invasive urban forest pest invades natural environments - Asian 
longhorned beetle in northeastern US hardwood forests. Canadian Journal of Forest Research, v. 41, n. 9, p. 1729-1742, 2011.

DOMINIAK, B. et al. Survey for sycamore lace bug Corythucha ciliata (Say) (Hemiptera: Tingidae) in New South Wales during 2007. General and Applied Entomology, v. 37, p. 27-30, 2008.

ELITH, J. et al. Novel methods improve prediction of species' distributions from occurrence data. Ecography, v. 29, n. 2, p. 129-151, 2006.

ELITH, J. et al. A statistical explanation of MaxEnt for ecologists. Diversity and Distribution, v. 17, n. 1, p. 43-57, 2011.

FICETOLA, G. F., THUILLER, W., MIAUD, C. Prediction and validation of the potential global distribution of a problematic alien invasive species the American bullfrog. Diversity and Distribution, v. 13, n. 4, p. 476-485, 2007.

GOOGLE MAPS - Google Maps. Disponível: $<$ http://maps.google.com/>. Acesso em: 19/09/2011. GROSSO-SILVA, J. M.; AGUIAR, A. Corythucha ciliata (Say, 1832) (Hemiptera, Tingidae), the nearctic Sycamore lace bug, found in Portugal. Boletín Sociedad Entomológica Aragonesa, n. 40, p. 366-366, 2007.

GUISAN, A., THUILLER, W. Predicting species distribution: offering more than simple habitat models. Ecology Letters, v. 8, n. 9, p. 993-1009, 2005.

GUISAN, A. et al. Using niche-based models to improve the sampling of rare species. Conservation Biology, v. 20, n. 2, p. 501-511, 2006.

JU, R.-T. et al. Spread of and damage by an exotic lacebug, Corythucha ciliata (Say, 1832) (Hemiptera: Tingidae), in China. Entomological News, v. 120, p. 409-414, 2009.

JU, R. T, WANG, F.; LI, B. Effects of temperature on the development and population growth of the sycamore lace bug, Corythucha ciliata. Journal of Insect Science, v. 11:16 available online: insectscience.org/11.162011, 2011

MACELJSKI, M. Current status of Corythucha ciliata in Europe. Bulletin OEPP/EPPO, v. 16, p. 621-624, 1986.

MARINI, M. A. et al. Major current and future gap of Brazilian reserves to protect Neotropical savanna birds. Biology Conservation. v. 142, n. 12, p. 3039-3050, 2009.

MATASCI, M.; GESSLER, C. Ein pilz bedroht die existenz der platane. Acta Veterinaria Hungarica, v. 45, p. 69-75, 1997.

PETERSON, A. T. Predicting the geography of species' invasions via ecological niche modeling. Quarterly Review of Biology, v. 78, n. 4, p. 419-433, 2003.

PETERSON, A. T.; VIEGLAIS, D. A. Predicting species invasions using ecological niche modeling: new approaches from bioinformatics attack a pressing problem. BioScience, v. 51, n. 5, p. 363-371, 2001.

PETERSON, A. T.; PAPES, M.; KLUZA, D. A. Predicting the potential invasive distributions of four alien plant species in North America. Weed Science, v. 51, n. 6, p. 863-868, 2003.

PHILLIPS, S. J.; DUDÍK, M. Modeling of species distributions with Maxent: new extensions and a comprehensive evaluation. Ecography, v. 31, n. 2, p. 161-175, 2008.

PHILLIPS, S. J.; DUDÍK, M.; SCHAPIRE; R. E. A Maximum Entropy Approach to Species Distribution Modeling. In: INTERNATIONAL CONFERENCE ON MACHINE LEARNING, 2004, Banff, Proceedings... Banff, 2004.

PHILLIPS, S. J.; ANDERSON, R. P.; SCHAPIRE, R. E. Maximum entropy modeling of species geographic distributions. Ecological Modelling, v. 190, n. 3-4, p. 231-259, 2006.

PIMENTEL, D. Biological Invasions: Economic and Environmental Costs of Alien Plant, Animal, and Microbe Species. $2^{\text {nd }} e d$. CRC Press, 2011.

PRADO, E. Presencia en Chile de Corythucha ciliata (Say) (Hemiptera: Heteroptera: Tingidae). Revista Chilena de Entomologia, v. 18, p. 53-55, 1990.

ROMANYK, N.; CADAHIA, D. Plagas de insectos en las masas forestales españolas. Madrid: MundiPrensa. 2003.

ROURA-PASCUAL, N. et al. Consensual predictions of potential distributional areas for invasive species: a case study of Argentine ants in the Iberian Peninsula. Biological Invasions, v. 11, p. 1017-1031, 2009.

SERVADEI, A. Tingide neartico comparso in Italia (Corythuca ciliata Say). Bollettino della Societa Entomologica Italiana, Genova, v. 96, p. 94-96, 1966.

SIMBERLOFF, D.; REJMÁNEK, M. Encyclopedia of biological invasions. Berkeley: University of California Press, 2011.

THOMAS, C. D. et al. Extinction risk from climate change. Nature, v. 427, p. 145-148, 2004.

THUILLER, W. et al. Climate change threats to plant diversity in Europe. Proceedings of the National Academy of Sciences of the United 
States of America, v. 102, p. 8245-8250, 2005a. THUILLER, W. et al. Niche-based modeling as a tool for predicting the risk of alien plant invasions at a global scale. Global Change Biology, v. 11, n. 12 , p. 2234-2250, 2005b.

TOKIHIRO, G.; TANAKA, K.; KONDO, K. Occurrence of the sycamore lace bug, Corythucha ciliata (Say) (Heteroptera: Tingidae) in Japan. Research Bulletin of the Plant Protection Service Japan, v. 39, p. 85-87, 2003.

VILLEMANT, C. et al. Predicting the invasion risk by the alien bee-hawking Yellow-legged hornet Vespa velutina nigrithorax across Europe and other continents with niche models. Biology Conservation, v. 144, n. 9, p. 2142-2150, 2011.

WILSON, K. A. et al. Sensitivity of conservation planning to different approaches to using predicted species distribution data. Biology Conservation, v. 122, n. 1, p. 99-112, 2005.

WORLDCLIM - Global Climate Data. Disponível: <http://www.worldclim.org/>. Acesso em: 20/09/2011. 